\title{
Facilitando o aprendizado de programação sob a ótica de metodologias ágeis
}

Title: Facilitating the programming from the perspective of agile methodologies

\author{
Vilson C. Gärtner \\ Mestrado em Computação Aplicada \\ UNISINOS \\ vilson@gartner.net.br
}

\author{
Sergio Crespo C S Pinto \\ Mestrado em Computação Aplicada \\ UNISINOS \\ crespo.sergio@gmail.com
}

\author{
Arthur Torgo Gómez \\ Mestrado em Computação Aplicada \\ UNISINOS \\ atgomezbr@gmail.com
}

Resumo Dentro das metodologias ágeis a ideia de programação em pares obedece de forma implícita esta teoria. A ideia deste trabalho é proporcionar que um conjunto maior de pessoas possam compartilhar o desenvolvimento de um código fonte de forma colaborativa e que fique registrado no ambiente o histórico das alterações e todo o processo intelectual que leva a mudanças em determinadas partes de um código fonte. Isto permite que uma relação $N-N$ possa ser estabelecida em um ambiente de desenvolvimento de software distribuído, potencializando a teoria do par mais capaz. O artigo apresenta uma ferramenta desenvolvida como um plug-in do NetBeans que incorpora várias funcionalidades de ambientes colaborativos para suporte a programação distribuída em tempo real.

Palavras-Chave: Programação em pares, Programação, Programação Distribuída

\begin{abstract}
The agile idea of pair programming implicitly obeys this theory. The idea of this paper is to provide that a larger set of people can share the development of a source code in a collaborative manner and that the history and the whole intellectual process that leads to changes in certain parts of a source code will be record in the environment. This allows a $N-N$ relationship can be established in a development environment for distributed software, leveraging the theory of the more capable peer. The article presents a tool developed as a NetBeans' plug-in, functionality that incorporates several of collaborative environments to support distributed programming in real time.
\end{abstract}

Keywords: Pair Programming, Programming, Distributed Programming 


\section{Introdução}

O aumento dos ambientes colaborativos em empresas produtoras de software desencadeia um novo perfil do aluno que entra nos cursos de computação: "o perfil de trabalhar em grupo". Para isto, é necessário aliar o uso de ferramentas colaborativas e teorias pedagógicas que reforcem a colaboração entre pares e acentuem a colaboração em atividade de desenvolvimento de software. Por outro lado, desenvolver software é uma atividade altamente intelectual e que exige troca de conhecimento constante entre as pessoas envolvidas. Tal necessidade por interação é evidenciada por algumas práticas de desenvolvimento, como a "programação em pares". É necessário, ainda, que os pares sejam permutados a cada nova atividade, de forma a disseminar o conhecimento adquirido entre os aprendizes.

Para [24], "o olho humano tem uma grande capacidade de não enxergar aquilo que não quer ver". Da mesma forma, um aluno iniciante pode ignorar completamente um erro gritante que seria rapidamente percebido por um observador mais experiente. Pesquisas realizadas pelos autores revelam que dois programadores juntos chegam a uma solução mais rápida e com maior qualidade do que dois programadores trabalhando separadamente na resolução de problemas.

[13] identifica que as iniciativas para viabilizar programação por pares distribuídos utilizam aplicações não orientadas para este fim. São utilizados: compartilhamento de desktop, e-mail, troca instantânea de mensagens, voz sobre IP, repositórios de arquivos compartilhados e videoconferência. Tais aplicações possuem objetivos específicos e não são orientadas à programação. Neste contexto, torna-se importante a existência de um ambiente integrado que viabilize diferentes formas de comunicação orientadas ao ensino de programação, por pares, de forma distribuída e colaborativa[13]. Neste sentido, este trabalho apresenta a ferramenta IdDE. Esta consiste em um ambiente colaborativo para auxílio à programação distribuída em pares. A sua principal contribuição está na possibilidade de utilização de ferramentas interativas que tornam mais eficiente o processo de colaboração. Além disso, do ponto de vista pedagógico, trabalhar em pares, valendo-se de um instrumento mediador, segundo o princípio sociocultural da teoria Vygotskyana, influi nas interações, pois, em geral, o indivíduo costuma explicitar suas estratégias durante a resolução de tarefas compartilhadas

\section{Groupware e sua fundamentação pedagógica}

Segundo [10], CSCW ou Groupware é definido como o estudo dos sistemas que integram o processamento de informações com atividades de comunicação, de forma a identificar como os grupos trabalham e como a tecnologia pode ajudá-los a resolver tarefas em comum, reforçando a importância da comunicação, colaboração e coordenação [10].

Conforme [12], a comunicação oportuniza a negociação e o estabelecimento de compromissos entre os participantes do grupo. Para utilizar o computador como ferramenta de comunicação, é necessário que o sistema ofereça suporte à interação entre as pessoas. Deve haver controle entre os estados, eventos e diálogos de cada participante. De acordo com o modelo, o emissor define a sua intenção (no nível mais abstrato de ideias) e a fórmula através de símbolos. Utiliza o computador para expressá-la numa mensagem e transmiti-la via canal de dados. Chegando ao destino, a mensagem é percebida e interpretada, de forma a modificar seus argumentos. As ferramentas de comunicação podem ser classificadas em dois tipos, podendo ser analisadas quanto ao tipo de resposta e quanto ao nível de aplicação [12].

- Quanto ao tempo de resposta: podem ser síncronas e assíncronas.

- Quanto ao nível de aplicação:

- Sistemas de mensagens - suportam a troca de mensagens de texto entre usuários;

- Editores multiusuários - onde códigos podem ser alterados por vários usuários;

- Decisão em grupo - oferecem mecanismos para tomada de decisão em grupo;

- Conferências - São em geral módulos com apoio de áudio e vídeo;

- Agentes Inteligentes - Sistemas de software autônomos;

- Sistemas de coordenação - permitem o controle e gerência das atividades do grupo.

Os autores estabelecem, ainda, um conjunto de funcionalidades importantes a sistemas de colaboração em tempo real (síncronas), conforme apresenta a Tabela I:

\begin{tabular}{c|c}
\hline Funcionalidade & \multicolumn{1}{|c}{ Definição } \\
\hline Ações & $\begin{array}{l}\text { O conjunto de objetos e ações, } \\
\text { realizadas e percebidas por to- }\end{array}$ \\
\hline
\end{tabular}




\begin{tabular}{|c|c|}
\hline Funcionalidade & Definição \\
\hline & dos. \\
\hline Janelas & $\begin{array}{l}\text { Áreas que estão compartilhadas e } \\
\text { sincronizadas por todos os parti- } \\
\text { cipantes on-line. }\end{array}$ \\
\hline Cursos & $\begin{array}{l}\text { Movimento do cursor é percebido e } \\
\text { disponibilizado a todos. }\end{array}$ \\
\hline Visão & $\begin{array}{l}\text { Uma representação visual ou mul- } \\
\text { timídia de parte do conteúdo com- } \\
\text { partilhado. }\end{array}$ \\
\hline Interação & $\begin{array}{l}\text { Ações percebidas de forma síncro- } \\
\text { na e assíncrona. }\end{array}$ \\
\hline Seção & Período de interação síncrona. \\
\hline Papéis & $\begin{array}{l}\text { Conjunto de privilégios, atribui- } \\
\text { ções ou responsabilidades de par- } \\
\text { ticipante do grupo. }\end{array}$ \\
\hline
\end{tabular}

Tabela 1: Funcionalidades de Sistemas de Colaboração Síncronas

\subsection{Desenvolvimento de Software Distribuído}

A tendência pelo desenvolvimento de software através de grupos geograficamente distribuídos é justificada, segundo [15], pela redução de custos e busca por mão de obra qualificada. Os autores citam fatores como necessidades competitivas e a globalização das operações corporativas como atenuantes dessas tendências. Evidências indicam que o desenvolvimento distribuído costuma consumir tempo maior, quando comparado ao realizado por equipes geograficamente centralizadas, identificando a comunicação e coordenação como principais causas para esse aumento de tempo [15].

\subsection{Programação em Pares Distribuída}

Vários estudos têm sido realizados com o objetivo de avaliar as diferenças apresentadas, quanto à produtividade e qualidade, entre a programação em pares distribuída e a realizada "lado a lado". Em [22] são citados como vantagens:

- Redução da necessidade de viagens;

- Os pares são forçados a manter registros eletrônicos de seus trabalhos e ideias;

- Os membros tendem a diminuir as conversas offtopic.

Schummer percebeu que os programadores devem estar aptos a se comunicar, ambos devem acompanhar o mesmo trecho de código, mas apenas um deve alterá-lo. A comunicação pode ocorrer por voz. Para os autores e coautores, o contato visual não é necessário [20][25].

\subsection{Teorias pedagógicas que fundamentam o modelo Groupware}

A interatividade está presente como elemento fundamental no processo de construção do conhecimento do indivíduo. De acordo com Piaget o conhecimento não está no sujeito nem no objeto, mas ele se constrói na interação do sujeito com o objeto. É na medida em que o sujeito interage (e, portanto age sobre e sofre ação do objeto) que ele vai produzindo sua capacidade de conhecer e vai produzindo também o próprio conhecimento [11]. Já Vygotsky aborda este processo do ponto de vista de interatividade entre o indivíduo e o meio social. $\mathrm{O}$ princípio sociocultural da teoria Vygotskyana nos traz um forte embasamento pedagógico para o trabalho em equipe, onde a importância da comunicação, colaboração e coordenação a fim de viabilizar o trabalho em grupo e obter melhores resultados, quando comparados ao trabalho realizado individualmente, é o elemento de sucesso.

Para Vygotsky, os processos mentais só podem ser entendidos se forem entendidos os instrumentos e os signos que mediam esses processos. Além disso, o desenvolvimento cognitivo do ser humano não pode ser entendido fora do contexto social e cultural em que este se produz. E para entender o desenvolvimento cognitivo do ser humano, bem como os instrumentos e signos que mediam esses processos, Vygotsky se apoia no método genético experimental. Compreende-se então que, para Vygotsky, o modelo histórico-social é esboçado nas estruturas de mediação instrumental e social, que por sua vez internalizam, no ser humano, estruturas que possibilitam a interpretação do movimento, quer pela passagem de ações realizadas no plano social, portanto interpsicológicas, quer pela passagem de ações internalizadas (intra-psicológicas). No caso de ferramentas de Groupware essas ações interpsicológicas e intra-psicológicas possuem uma dimensão não linear o que possibilita ao indivíduo uma postura exploratória maior. Desse modo as interações entre o grupo potencializam construções de conhecimento mais autônomos e criativos [18]. Trabalhar em pares, valendo-se de um instrumento mediador, influi nas interações, pois, em geral, o indivíduo costuma explicitar suas estratégias durante a resolução de tarefas compartilhadas.

\section{Trabalhos Relacionados}

Nesta seção serão apresentados alguns trabalhos pertinentes ao estudo.

\subsection{VIMEE}

É um ambiente para auxiliar a comunicação e a tomada de decisão em equipe. Possibilita uma comunicação síncrona, explícita e formal em ambientes DSDE (Distributed Software Development Environment) [23]. A comunicação entre os participantes ocorre de maneira clara e explícita, e é baseada em procedimentos formais que guiam o andamento das atividades, indicando quem está habilitado a executar algo. Algumas de suas características são: 
- Agendamento de reuniões, com aviso aos participantes;

- Permite a troca síncrona de mensagens de texto;

- Não possui limites quanto ao número de participantes;

- Permite a visualização de artefatos do projeto;

- Permite o compartilhamento de documentos e imagens;

- Possibilita o armazenamento de documentos gerados numa reunião;

- Possibilita a realização de votações, para tomada de decisão.

Para organizar o fluxo dos procedimentos, o VIMEE permite a coordenação das reuniões através do papel de um mediador. A interface do mediador difere da interface dos demais usuários, e tem por objetivo possibilitar: o início e término de uma reunião, bloquear ou liberar algum participante, controlar as votações e enviar mensagens. Nessa interface, também é controlado qual usuário, previamente inscrito, pode se manifestar. Aliado a isso, possibilita a liberação do acesso aos artefatos do projeto, além de poder tornar visível a existência de documentos e imagens para os demais participantes.

\subsection{RemotePP}

O RemotePP é um ambiente cooperativo de desenvolvimento com o objetivo de dar suporte à programação em pares distribuídos [2]. Como o seu objetivo é voltado para a programação em pares, a sessão somente pode ser estabelecida entre dois programadores, um local e outro remoto, desde que esteja dentro da mesma rede. O ambiente possui uma série de ferramentas, a seguir descritas:

- Edição de código-fonte: este módulo permite a edição de programas de forma colaborativa, porém, não simultâneo, ou seja, somente um programador pode alterar o código por vez. Caso a sessão não tenha sido estabelecida com o outro usuário, a edição acontece somente de forma local;

- Quadro branco: funcionalmente similar ao módulo de edição de código-fonte, este módulo permite que ambos os programadores, colaborativamente, editem desenhos e diagramas. Neste caso, a edição pode ser feita simultaneamente por ambos programadores;

- Chat: permite a troca de mensagens instantâneas entre os dois usuários;

- Vídeo: este módulo permite que os programadores realizem videoconferência. Para isso, é necessário que exista uma webcam instalada e configurada nos computador;

- Voz: possibilita que os programadores se comuniquem por áudio, utilizando o microfone acoplado ao computador;

- Ferramenta externa: permite que um programa externo seja executado, a exemplo de um compilador. As mensagens de saída, oriundas da execução do programa externo, serão exibidas para ambos os programadores.

\subsection{CollabEd}

Trata-se de um editor desenvolvido sobre uma plataforma de colaboração, segundo [17]. O design da plataforma compreende três categorias essenciais: conexão, manutenção e replay das interações. A arquitetura do aplicativo é baseada no modelo cliente/servidor. Tanto o servidor quanto o cliente são desenvolvidos na linguagem Java e, assim, são multiplataformas. Além de ser executado como uma aplicação independente, o CollabEd pode ser acoplado a editores de texto já existentes, através plugins. Já existem plugins disponibilizados para os editores Netbeans, Eclipse e JEdit. Neste aplicativo, o servidor é o encarregado de manter o histórico das alterações efetuadas nos clientes. Graças a este histórico, mesmo que os clientes mantenham as alterações em um buffer, as mesmas serão aplicadas corretamente pelo servidor, que se encarregará de atualizar a posição onde as alterações devem acontecer no documento remoto.

\subsection{Sangam}

O Sangam foi desenvolvido por [16] na forma de um plug-in para o ambiente de desenvolvimento Eclipse. A ferramenta oferece sincronização de código-fonte entre mais de dois programadores. O Sangam não bloqueia o texto durante uma sessão. A arquitetura do aplicativo utiliza três componentes principais:

- Interceptador de eventos - Monitora o ambiente a fim de capturar todas as ações que o usuário (driver) realiza no editor de código Java da aplicação;

- Servidor de mensagens - É responsável pelo envio das mensagens ao navigator;

- Reprodutor de eventos - Realiza as ações recebidas pelo servidor de mensagem de modo a reproduzi-las no computador do navigator.

\subsection{Flecse}

O Flecse atua como um conjunto de ferramentas colaborativas e aplicáveis a todo o ciclo de vida de desenvolvimento de software [8]. A interface de todas as aplicações é baseada em linha de comando e console texto, com as seguintes características: 
- O controle de versão: permite controlar a versão do programa;

- MShell: é um interpretador de comandos colaborativo;

- Teleconf: permite gravação, transmissão e execução de uma conversação por áudio;

- MDebug: possibilita a depuração de programas de forma compartilhada;

- CSI: é um mecanismo de inspeção de código assíncrono e síncrono;

\section{Um Ambiente de Suporte ao Desen- volvimento Colaborativo de Softwa-}

re

Esta seção apresenta o IdDE, um ambiente que oferece apoio ao processo colaborativo de programação de $\mathrm{N}$ :N usuários, mostrando de forma detalhada, o ambiente, suas ferramentas, sua arquitetura e tecnologias utilizadas. O IdDE esta em http://idde.vgdata.net/ para ftp.

\subsection{Introdução}

A colaboração possui um papel fundamental no desenvolvimento de software e uma organização terá mais benefícios das tecnologias colaborativas se utilizar um conjunto delas: mensagem instantânea, videoconferência, serviços de áudio de qualidade, repositórios integrados e ambientes de compartilhados [6]. Sob a perspectiva de um programador, um Ambiente Integrado de Desenvolvimento (IDE) possibilita a escrita de código e fornece uma série de funcionalidades importantes para o processo de aprendizagem de programação com enfoque colaborativo. O IdDE permite que a comunicação via áudio ocorra entre usuários do ambiente e dispositivos de telefonia convencional, telefonia móvel, telefones IP, ATAs e smartphones. Já a edição compartilhada poderá ocorrer entre aplicativos que implementam o protocolo de comunicação e negociação do IdDE. O chat, por sua vez, poderá ser utilizado em dispositivos que possuam softwares que utilizem o mesmo protocolo de comunicação adotado pelo ambiente.

\subsection{Tecnologias utilizadas}

As seguintes tecnologias foram utilizadas no desenvolvimento do IdDE: Java como linguagem de programação, NetBeans como IDE de desenvolvimento, protocolo $\mathrm{XMPP}^{1}$ (eXtensible Messaging and Presence Protocol) e protocolo SIP $^{2}$ (Session Initiation Protocol). A seguir são

\footnotetext{
${ }^{1} \mathrm{http}: / / \mathrm{xmpp}$. org
}

2 ://www.ietf.org/rfc/rfc3261.txt descritas as tecnologias.

- Linguagem de Programação Java: A escolha da linguagem de programação Java tem como objetivo possibilitar que o ambiente seja executado nas mais variadas plataformas operacionais, além de ser multiplataforma, é uma linguagem largamente utilizada e difundida;

- Ambiente Netbeans: Este ambiente oferece todas as funcionalidades necessárias para a edição de código fonte, além de disponibilizar inúmeras outras ferramentas, a exemplo de: formatação de código, dicas e sugestões de códigos automáticos, compilação, depuração, criador visual de formulários, suporte à internacionalização e suporte a diversas linguagens de programação;

- Protocolo XMPP: Este protocolo foi escolhido por ser um protocolo aberto e largamente utilizado para a comunicação. A tecnologia XMPP utiliza uma arquitetura cliente/servidor descentralizada, similar às arquiteturas utilizadas nas redes WWW e de e-mail [19]. Exemplos bastante conhecidos de seu uso são o Google Talk e Facebook;

- Protocolo SIP: O protocolo SIP é utilizado em comunicações do tipo VoIP. Segundo [9], SIP é um protocolo que controla a negociação, modificação e encerramento de uma sessão multimídia interativa. As sessões multimídia podem ser de áudio, vídeo, chat ou mesmo sessões de jogos [21].

\subsection{Arquitetura do Ambiente IdDE}

O Ambiente foi projetado para garantir uma arquitetura modular e foi desenvolvido utilizando a estrutura do Netbeans. Desta forma, o IdDE comporta-se como um módulo (ou plugin) do NetBeans.

A Figura 1 mostra de forma detalhada a arquitetura do ambiente. A interface e funções de interação com o usuário foram criadas independentes do IDE NetBeans, entretanto, são integradas ao mesmo através de sua arquitetura de plugins. Isso possibilita que o IdDE também possa ser integrado a outros IDEs, bastando para isso criar a camada de plugin (na figura, representado por "Interface"). Essa camada é a única parte que possui alguma dependência do Netbeans. Dessa forma, a interação do usuário com o ambiente acontece através das opções disponibilizadas pelo IdDE, e a interação deste com os editores será através da arquitetura de plugins dos mesmos.

Analisando a arquitetura do IdDE, é possível observar que as únicas funcionalidades do IdDE que estão diretamente vinculadas à camada de plugins do Netbeans são a edição compartilhada e a camada de interface, que estão relacionadas às janelas Principal e Controle de Sessões de 
edição. Essas janelas são criadas quando o módulo é carregado no Netbeans, o que ocorre durante a inicialização deste aplicativo.

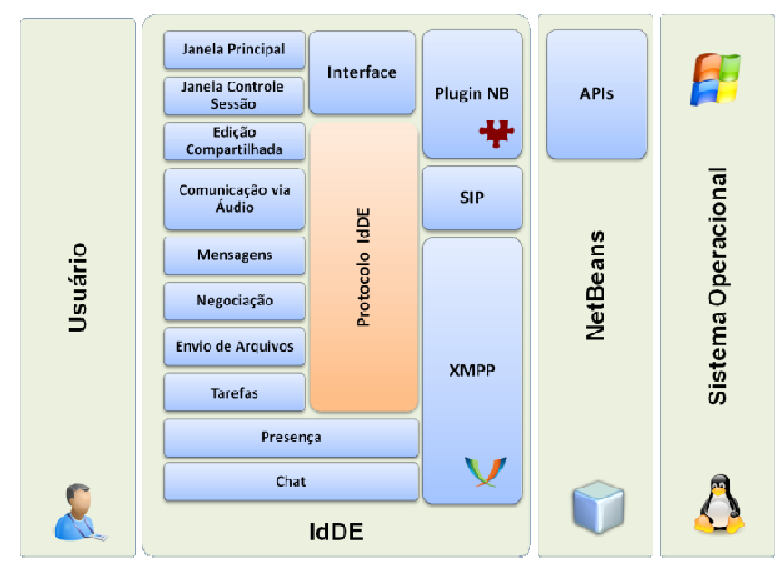

Figura 1: Arquitetura do ambiente

Pode-se verificar também que as funções acionadas pelo usuário fazem uso do Protocolo IdDE. Isso se deve ao fato de que o ambiente envia mensagens de negociação para o ambiente remoto, solicitando ou informando a ação executada pelo usuário local e que deve ser replicada para o usuário remoto. Desta forma, para que um aplicativo possa se comunicar com o IdDE, deve implementar, obrigatoriamente, esse mesmo protocolo. Por fim, a figura mostra que as funcionalidades de presença e chat interagem diretamente através de XMPP, não havendo a troca de mensagens ou utilização do protocolo IdDE. Isso permite que o IdDE se comunique com qualquer aplicativo que suporte o protocolo XMPP.

A Figura 2 representa a visão lógica da organização do sistema e suas ferramentas. No diagrama pode-se observar que os pacotes de classes foram organizados de forma a refletir a arquitetura modular apresentada na Figura 1. O nome de cada pacote faz referência à funcionalidade que implementa, e o detalhamento dessas funcionalidades é apresentado na Tabela II. Para implementar a comunicação no IdDE, foram utilizadas as bibliotecas: Smack $^{1}$ para a comunicação XMPP, Peers ${ }^{2}$ para comunicação SIP, além de partes de código do projeto Shortalk $^{3}$. A estruturação do código, conforme discutido anteriormente, foi feita de acordo com os módulos do aplicativo.

\footnotetext{
${ }^{1} \mathrm{http}: / / \mathrm{www}$.igniterealtime.org/projects/smack

${ }^{2} \mathrm{http} / / /$ peers.sourceforge.net

${ }^{3}$ http://code.google.com/p/shortalk
}

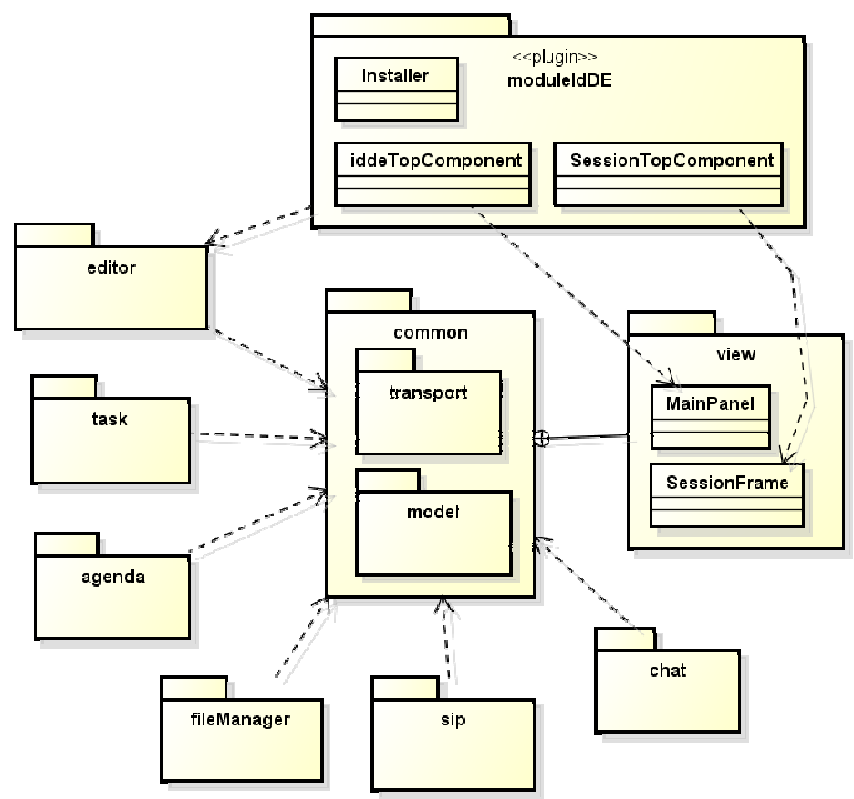

Figura 2: Estrutura de pacotes de classes do IdDE

\begin{tabular}{|c|c|}
\hline Pacote & Funcionalidade \\
\hline editor & $\begin{array}{l}\text { Possui as classes } \\
\text { responsáveis pela } \\
\text { implementação da edição } \\
\text { compartilhada e simultânea. }\end{array}$ \\
\hline task & $\begin{array}{l}\text { Neste pacote estão as } \\
\text { classes responsáveis pelo } \\
\text { gerenciamento de tarefas. }\end{array}$ \\
\hline agenda & $\begin{array}{l}\text { Agrega as classes } \\
\text { relacionadas à agenda de } \\
\text { compromissos. }\end{array}$ \\
\hline filemanager & $\begin{array}{l}\text { Classes responsáveis pela } \\
\text { transferência de arquivos } \\
\text { entre os usuários. }\end{array}$ \\
\hline sip & $\begin{array}{l}\text { Possui as classes que } \\
\text { implementam a comunicação } \\
\text { via áudio. }\end{array}$ \\
\hline common & $\begin{array}{l}\text { Este pacote agrega } \\
\text { funcionalidades comuns, } \\
\text { necessárias aos demais } \\
\text { pacotes. Este pacote possui } \\
\text { os subpacotes view, } \\
\text { transport e model. }\end{array}$ \\
\hline common: :view & $\begin{array}{l}\text { Neste pacote estão } \\
\text { localizadas as classes } \\
\text { responsáveis pela } \\
\text { implementação da tela } \\
\text { principal e de configuração } \\
\text { do IdDE (MainPanel), e da } \\
\text { tela com informações das } \\
\text { sessões de edição } \\
\text { colaborativa ativas } \\
\text { (SessionFrame). }\end{array}$ \\
\hline common: :transport & $\begin{array}{l}\text { Agrega as classes de } \\
\text { definição de mensagens do } \\
\text { protocolo IdDE, além de } \\
\text { criação e interpretação das } \\
\text { mensagens desse protocolo. }\end{array}$ \\
\hline common: :model & $\begin{array}{l}\text { Possui as classes que } \\
\text { implementam as } \\
\text { funcionalidades relacionadas }\end{array}$ \\
\hline
\end{tabular}




\begin{tabular}{l|l}
\hline \multicolumn{1}{c|}{ Pacote } & \multicolumn{1}{c}{ Funcionalidade } \\
\hline moduleIdDE & $\begin{array}{l}\text { ao XMPP, como conexão, envio } \\
\text { de mensagens e presença. }\end{array}$ \\
\hline & Neste pacote ficam \\
localizadas as classes \\
responsáveis pela integração \\
do IdDE ao Netbeans e pela \\
interação com o documento de \\
edição de código fonte. As \\
principais classes são: \\
Installer, responsável pela \\
“instalação e ativação do \\
módulo no Netbeans; \\
IddeTopComponent, \\
responsável pela \\
apresentação da tela \\
principal do IdDE no \\
Netbeans; e \\
SessionTopComponent, \\
responsável por apresentar a \\
tela com informações das \\
sessões de edição \\
compartilhada existentes.
\end{tabular}

Tabela2: Pacotes e suas funcionalidades do [5], deve-se possibilitar a análise e revisão síncrona online do código. Aliado a isso, a ferramenta também contribuirá na aprendizagem de desenvolvedores iniciantes, possibilitando que estes acompanhem a programação de usuários mais experientes.

A arquitetura da ferramenta de colaboração pode ser vista na Figura 3. O pacote moduleIdDE é o responsável pelo integração do ambiente ao Netbeans e possui as classes encarregadas pelo monitoramento dos eventos no documento. A classe DocumentChangesListener monitora as alterações (inclusão e exclusão de caracteres) que ocorrem no documento e a classe DocumentCaretListener é a responsável por fazer o monitoramento das alterações na posição do cursor. Também neste pacote estão as classes: EditAnnotation, responsável pela criação das características visuais no ambiente (marcações de alterações feitas e seus respectivos autores), e a classe CaretPosAnnotation responsável pelas marcações visuais relativas à posição do cursor dos usuários remotos.

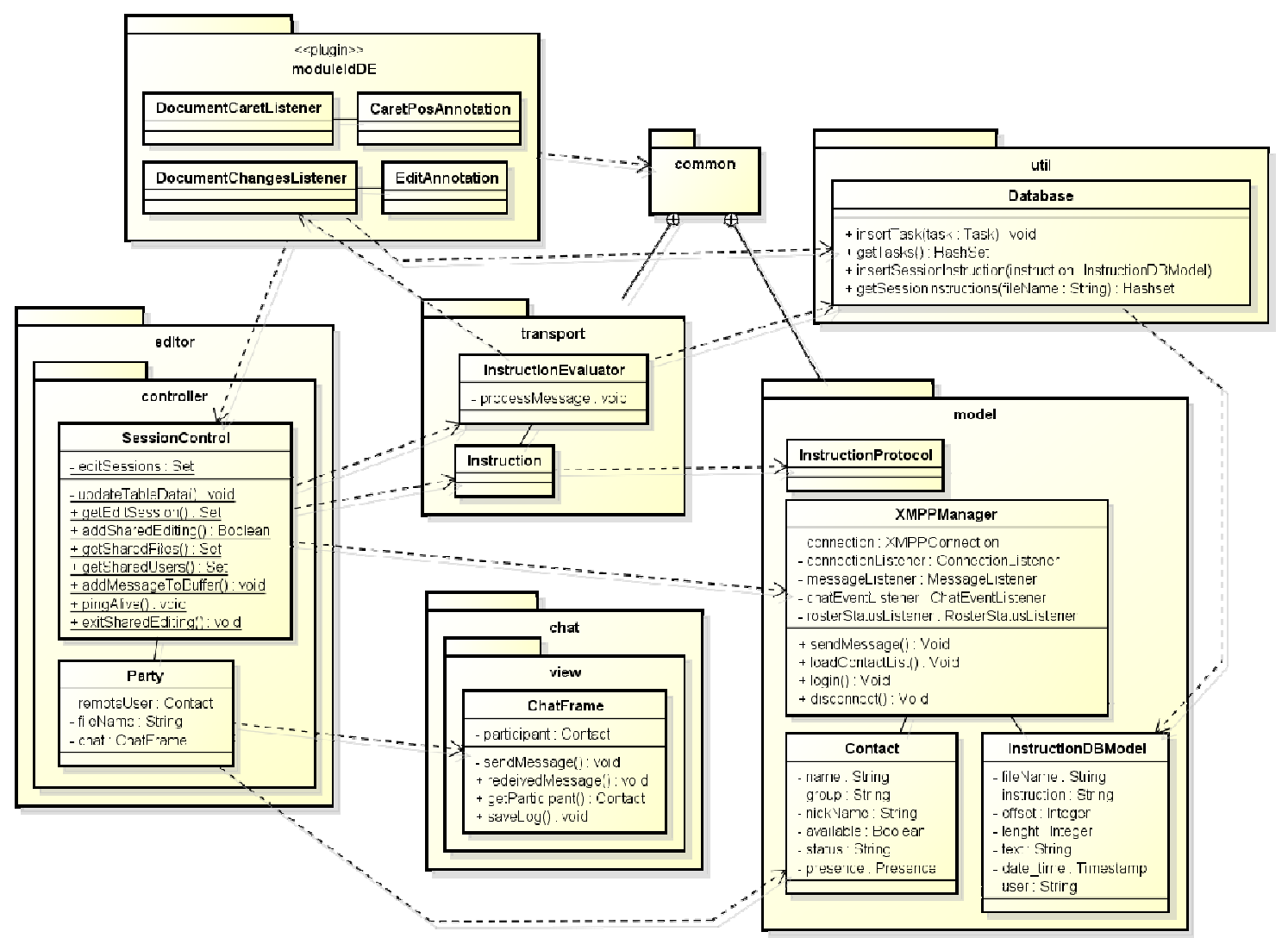

Figura 3: Arquitetura da ferramenta de edição colaborativa

A ferramenta de edição colaborativa do ambiente IdDE permite que vários usuários acessem e modifiquem o mesmo código fonte, simultaneamente. A edição em tempo real tem papel fundamental no desenvolvimento distribuído, auxiliando para que se tenha um código padronizado, otimizado e de melhor qualidade final. Segun-
A classe SessionControl do pacote editor::controller é responsável por controlar as sessões de edição de documentos, estabelecidas entre dois (ou mais) usuários. Entre os métodos importantes dessa classe estão: getEditSession, responsável por iniciar uma nova sessão de edição colaborativa; addSharedEditing, responsável por incluir 
um novo usuário a uma sessão; getSharedFiles, método utilizado para retornar os arquivos que estão numa sessão de edição; getSharedUsers, utilizado para retornar os usuários que participam de uma sessão; pingAlive, utilizado para verificar se os usuários continuam na sessão; exitSharedEditing, executado quando um usuário sai de uma sessão compartilhada; e addMessageToBuffer, método utilizado para enviar uma mensagem do protocolo para os demais usuários da sessão. As informações das sessões existentes são mantidas no atributo editSessions.

\subsection{O Ambiente IdDE}

As interfaces desenvolvidas no IdDE foram encapsuladas no sistema de janelas do Netbeans, através da utilização de suas APIs. Na Figura 4 essas interfaces são identificadas pelas classes MainPanel e SessionFrame do pacote common::view. Essas janelas são criadas automaticamente quando o plugin do IdDE é carregado, o que ocorre durante a inicialização do Netbeans.

Na Figura 4 estão destacadas ambas as janelas do IdDE: a janela principal (A) na qual é feita a configuração do aplicativo e mostrados os usuários conectados; e a janela de sessões (B) que exibe as sessões de edição simultânea estabelecidas. Como está integrado ao Netbeans, o IdDE dispõe de todos os mecanismos deste ambiente, como por exemplo, a possibilidade de minimizar ou ocultar as janelas, o que amplia o espaço de edição.

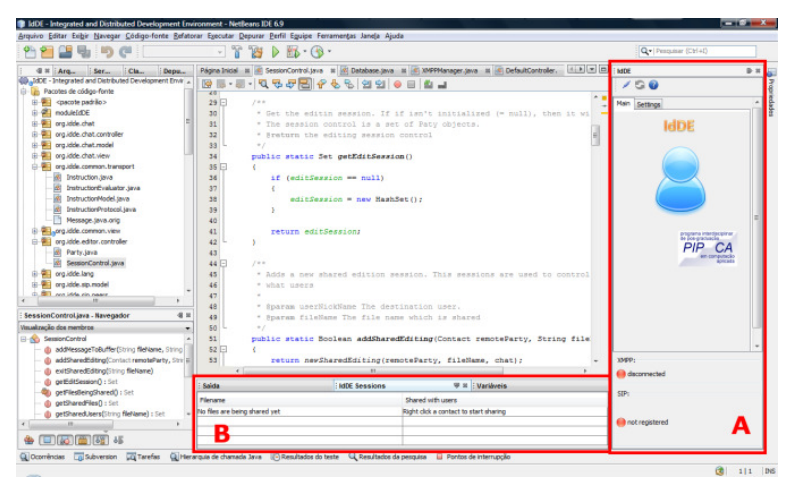

Figura 4: Integração do IdDE no Netbeans

\subsubsection{Edição Colaborativa no Ambiente grá- fico}

A ferramenta de edição colaborativa é a que mais utiliza a troca de mensagens entre os ambientes. Após estabelecer a comunicação entre os ambientes e adicionar os listeners necessários aos documentos, todas as modificações feitas por um usuário serão enviadas aos demais usuários participantes da sessão e serão replicadas naqueles ambientes. Quando o ambiente detecta que um usuário fez uma modificação num documento, o primeiro passo é verificar se este documento está em uma sessão de edição colaborativa. Caso não esteja, o processo é concluído e nenhuma ação é tomada. Entretanto, se ele estiver sendo compartilhado, é necessário avaliar o tipo de alteração ocorrida e compor a instrução apropriada para os demais usuários participantes.

As modificações no estado podem ser alteração de texto, como a inclusão ou exclusão de caracteres, ou a mudança na posição do cursor. Quando ocorrer uma mudança na posição do cursor, o ambiente verifica o novo posicionamento e envia uma mensagem aos ambientes remotos, para que estes atualizem essa informação. Quando ocorrer a alteração de texto, o IdDE verifica se houve inclusão ou exclusão de texto. Em caso de exclusão de um trecho de código, o único procedimento a ser executado é a criação e o envio da respectiva mensagem para os ambientes remotos informando onde ocorreu a remoção. No caso de inclusão, são informados, além da posição, os caracteres que devem ser adicionados.

Quando recebe instruções de outros usuários, além de efetuar a alteração no código, o IdDE adiciona marcações visuais ao ambiente, como pode ser observado na Figura 5 , que permitem localizar e identificar tanto as alterações quanto seus autores. Essas informações são importantes, pois auxiliam o usuário na análise e revisão síncrona online do código alterado, o que é essencial, segundo [5]. Estes recursos visuais utilizam-se das APIs nativas do Netbeans. Dessa forma, em uma adaptação do plugin para outro IDE, tais funcionalidades deverão ser reescritas utilizando as funcionalidades disponibilizadas por aqueles ambientes. A Figura 5 mostra detalhadamente esses elementos.

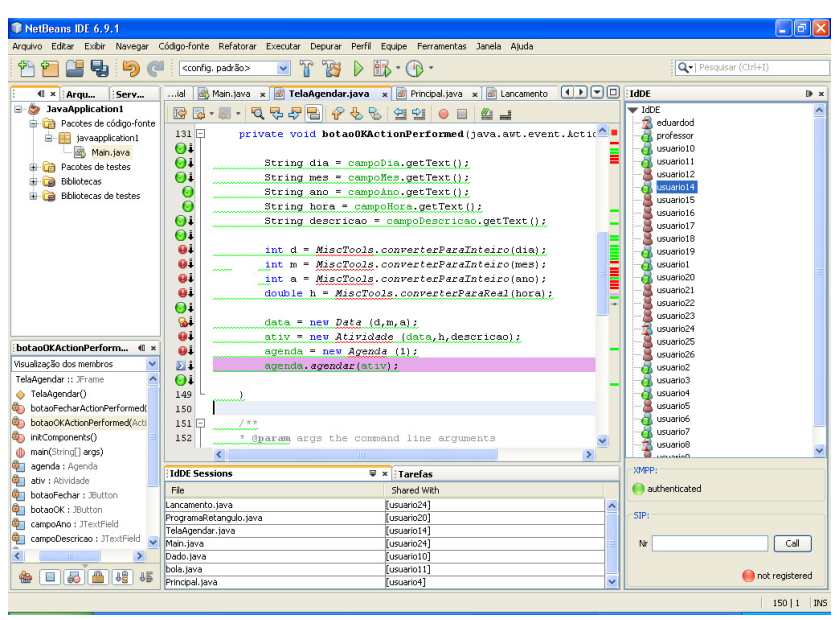

Figura 5: Alteração de código

As marcações visuais adicionadas pelo IdDE ao Netbeans são a marcação do texto alterado e a indicação da posição do cursor do usuário remoto. 


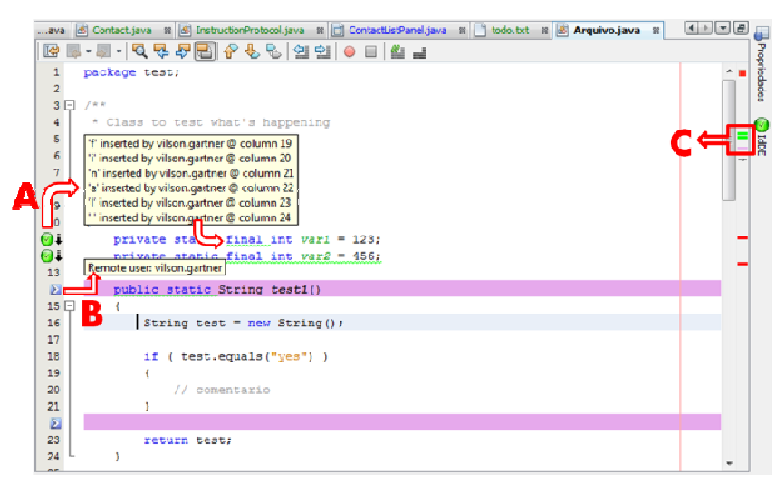

Figura 6: Marcação de código e posicionamento de cursor

As marcações visuais adicionadas pelo IdDE ao Netbeans são a marcação do texto alterado e a indicação da posição do cursor do usuário remoto. A Figura 6 mostra detalhadamente essas marcações.

Quando o ambiente recebe uma mensagem de alteração de código de um usuário remoto, essas modificações são imediatamente aplicadas no código local e marcadas através da utilização de uma sublinha ondulada em verde. Além disso, as linhas que sofreram alterações são marcadas com um ícone com a forma de um círculo verde (Figura 6-A). Quando o usuário desloca o ponteiro do mouse sobre este ícone, são exibidas informações detalhadas como dados inseridos, coluna e autor, como mostra a imagem.

Outra marcação importante adicionada ao documento é a indicação da linha na qual está posicionado o cursor do usuário remoto. Essa marcação é representada por um ícone azul com uma seta (Figura 6-B). Como é possível que vários usuários editem o documento ao mesmo tempo, para saber qual a posição de um determinado usuário, basta que se desloque o ponteiro do mouse sobre o ícone e o ambiente apresentará o nome do usuário, conforme pode ser observado na imagem. Quando o usuário remoto deslocar o cursor em seu ambiente, essa informação é igualmente atualizada no ambiente local. Nos estudos de caso realizados, pode-se perceber que essa característica contribui para o aumento da interatividade e experiência do usuário.

Em casos nos quais o código fonte é muito extenso, são criadas barras de rolagem no documento, da mesma forma como ocorre em outros editores. Nestas situações, pode acontecer de o usuário remoto fazer alterações na parte do texto que não está visível para o usuário local. Assim, para que este tenha ciência das alterações que estão sendo feitas no código, são criadas também as marcações indicadas pela Figura 6 (C). Nestas marcações, as cores identificam o tipo de ocorrência e, deslocando o mouse sobre as mesmas, são exibidas informações detalhadas, a exemplo do que ocorre quando se desloca o mouse sobre os ícones. Adicionalmente, é possível clicar nessas marcações, fazendo com que o editor traga para a área visível o trecho de código com a respectiva ocorrência.

Considerando que, teoricamente, é possível estabelecer um número ilimitado de sessões, é importante proporcionar ao usuário a informação de quais arquivos estão sendo compartilhados e quais usuários estão participando dessas sessões. Caso o usuário participe de muitas sessões de edição colaborativa, essas informações passam a ser ainda mais importantes.

Para auxiliar o usuário nesse aspecto, foi criada uma janela específica para manter as informações das sessões de edição colaborativa existentes, exibida Figura 42. Essa janela é composta por uma tabela, contendo o nome do arquivo compartilhado e os usuários participantes da sessão. Toda vez que uma nova sessão de edição colaborativa é estabelecida, uma nova linha é adicionada à tabela. Da mesma forma, quando um novo usuário passa a fazer parte da sessão, seu nome é adicionado àquela informação. A figura 7 apresenta a interface para gerenciamento de informações,

\begin{tabular}{|c|c|c|}
\hline IdDE Sessions & $\nabla \times$ Tarefas & \\
\hline File & Shared With & \\
\hline Lancamento.java & [usuario24] & 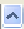 \\
\hline ProgramaRetangulo.jawa & [usuario20] & \\
\hline Telamggendar,java & [ussuario 14] & \\
\hline Main,jaya & [usuario24] & \\
\hline Dado.java & [usuario 10] & \\
\hline bola.java & [usuario 1] & \\
\hline \multirow[t]{7}{*}{ Principal,jaya } & \multirow{2}{*}{ File: bola.java } & \\
\hline & & \\
\hline & Send chat message to all users & \\
\hline & Lẹave this editing session & \\
\hline & Show all changes made on this file & \\
\hline & Send my version to remote (All) & \\
\hline & Verify if users are still connected & \\
\hline
\end{tabular}

Figura 7: Janela de controle de seção e menu de contexto

Além de exibir as informações sobre os arquivos e usuários das sessões, essa janela também dá acesso a outras funcionalidades relacionadas à sessão. Clicando com o botão direito sobre uma linha da tabela, é apresentado um menu de contexto, com as funções a seguir descritas:

- "Send chat message to all users": através desta opção pode-se enviar uma mensagem de texto para todos os usuários participantes da sessão;

- "Leave this editing session": esta opção deve ser utilizada quando se deseja sair de uma sessão de edição colaborativa. Outras formas de sair de sessões são: finalizar a conexão com o servidor XMPP, fechar o documento ou ainda fechar o Netbeans. Em todos estes casos o ambiente envia uma mensagem alertando os ambientes remotos que o usuário está deixando a sessão.

- "Show all changes made on this file": acessando esta opção é apresentada ao usuário uma janela com todas as alterações feitas na sessão de edição 
selecionada. Os dados constantes nessa tabela são: código do protocolo indicando o tipo de alteração ocorrida (inclusão ou exclusão), posição onde a modificação aconteceu, a informação incluída, o autor e momento da alteração. Quando se trata da exclusão de informações, o campo de informação incluída fica em branco.

As informações da tabela podem ser ordenadas pressionando-se o título da coluna. Dessa forma, as informações podem ser agrupadas permitindo, por exemplo, uma análise para verificar as alterações feitas por um mesmo autor, tipo de alteração ou seguindo a ordem que as alterações ocorreram.

Além de fornecer o histórico das alterações efetuadas, essa funcionalidade permite que se efetuem outras análises posteriores como, por exemplo, avaliar a lógica de programação utilizada por um usuário na resolução de um problema ou implementação de uma nova funcionalidade a um programa. Além disso, com base nessas informações é possível implementar uma ferramenta que permita a execução passo-a-passo de todas as alterações que ocorreram num arquivo, podendo-se simular todo o processo desde o princípio.

- "Send my version to remote (All)": esta opção é utilizada para enviar o código do ambiente local para os demais participantes da sessão. Esta opção era especialmente útil quando o ambiente ainda não implementava o controle da ordem das mensagens;

- "Verify if users are still connected": através desta opção é possível verificar se todos os usuários participantes da sessão continuam conectados. Caso um usuário remoto tenha sido desconectado de forma abrupta, ele permanecerá na lista de usuários da sessão e o ambiente continuará a enviar as mensagens de alterações. Assim, esta opção auxilia a eliminar usuários que não estiverem mais conectados.

\subsubsection{Controle de versões durante a edição colaborativa no ambiente IdDE}

No contexto do IdDE, as sessões de edição colaborativa permitem o acompanhamento das alterações em tempo real, enquanto que o uso da ferramenta de controle de versões mantêm uma visão macro das alterações efetuadas ao longo do tempo. O controle de versões está integrada diretamente no Netbeans e, dessa forma, todas as alterações feitas num arquivo automaticamente ficam registradas num repositório local, controlado pelo Netbeans. Além disso, esse IDE também possui suporte e integração com outros repositórios como: Subversion, Mercurial e CVS.

\subsubsection{Gerenciamento de Tarefas}

Através da ferramenta de controle de tarefas do IdDE, será possível atribuir tarefas aos programadores, acompanhar sua execução e progresso, permitindo que os programadores adicionem observações às mesmas.

No modelo proposto pelo ambiente, as tarefas não ficam armazenadas num banco de dados centralizado, mas sim, distribuídas de forma descentralizada no banco de dados de cada usuário. Dessa forma, quando um usuário quiser saber o detalhamento das tarefas de outro, basta acessar a opção apropriada no ambiente.

Em trabalhos futuros, pretende-se estudar a viabilidade da integração desse módulo a ferramentas online como, por exemplo, o Google Calendar. Essa integração possibilitaria que outros stakeholders que não possuam acesso ao ambiente de desenvolvimento possam acessar as informações relativas às tarefas.

As funcionalidades de gerenciamento também são acessadas através do menu de contexto na lista de contatos, conforme mostra a Figura 8. No menu, existem três opções disponíveis, descritas a seguir:

- Add task for this user: esta opção deve ser utilizada para adicionar uma nova tarefa para o usuário.

- View user's tasks: Esta opção deve ser utilizada para ver as tarefas existentes para o usuário remoto. Nessas solicitações, o ambiente remoto responde com uma mensagem de chat, listando as tarefas do usuário;

- Open task manager: Essa opção apresenta uma tela com as tarefas do usuário local. Através dessa tela é possível alterar informações.

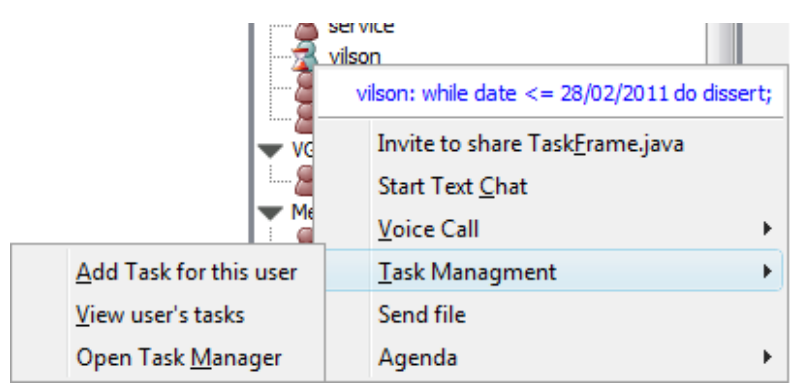

Figura 8: Menu de contexto e opções relacionadas a tarefas

\section{Estudo de Casos}

No total foram realizados quatro estudos de casos em grupos heterogêneos, abrangendo profissionais que trabalham no desenvolvimento de software, e professores e acadêmicos de cursos de graduação da área de tecnologia 
da informação. Neste trabalho são apresentados unicamente os três experimentos executados no ambiente acadêmico.

\subsection{Cenário 1: Estudantes Universitários}

O primeiro estudo de caso foi realizado com alunos de uma turma de graduação do Centro Universitário UNIVATES, na cidade de Lajeado/RS. A turma era composta por alunos dos cursos de Sistemas de Informação, Engenharia da Computação e Análise de Sistemas, todos cursando a disciplina de Programação Orientada a Objetos.

Os testes foram realizados num total de duas aulas, no mês de Dezembro de 2010. Alguns alunos participantes já atuavam na área de desenvolvimento de sistemas, enquanto que outros somente estudavam ou eram profissionais de outras áreas. As ferramentas testadas foram edição colaborativa e comunicação via chat.

\subsubsection{O processo utilizado}

Os testes deste cenário foram realizados num dos laboratórios da instituição, com um total de 23 participantes. Antes do início dos testes, foi feita uma apresentação da ferramenta, descrevendo suas características e seus objetivos. Em seguida, os próprios alunos executaram a instalação do plugin no Netbeans, que já é utilizado normalmente nas disciplinas de programação. O sistema operacional utilizado para estes testes foi o Windows XP. Para a realização dos testes, foi utilizado um servidor XMPP interno (OpenFire ${ }^{1}$ ), visto que as políticas de segurança da instituição não permitiam a utilização de servidores externos. As ferramentas testadas neste cenário foram edição simultânea e comunicação através de mensagens instantâneas. A comunicação via áudio não foi possível em virtude do bloqueio das portas na rede da instituição.

Para a realização dos testes e análise do comportamento da ferramenta, os alunos foram instruídos a compartilharem arquivos com o professor, o qual acompanhava, a partir de sua mesa, as modificações feitas nos documentos. Além disso, o professor também colaborava com os alunos através da escrita de código diretamente no arquivo compartilhado.

A comunicação entre os alunos e professor, sempre que possível, ocorreu através da ferramenta de chat do ambiente. Todavia, foi possível perceber que, apesar da praticidade, os alunos com menos conhecimento de programação se sentiam mais confortáveis com a presença do professor ao seu lado.

Durante os testes realizados neste cenário, a ferramenta não apresentou nenhum tipo de problema. À medida que os alunos alteravam os códigos dos programas, eram

\footnotetext{
${ }^{1} \mathrm{http}: / / \mathrm{w}$ ww.igniterealtime.org/projects/openfire/
}

feitas comparações com a versão existente na máquina do professor, para verificar a ocorrência de alguma discrepância entre as duas versões.

\subsubsection{Análise dos resultados}

$\mathrm{Na}$ avaliação dos participantes, a ferramenta de chat se mostrou útil para todos eles. Alguns consideraram importante a possibilidade de poder efetuar a tradução das mensagens. Igualmente, para todos os respondentes a ferramenta auxiliou no processo de edição compartilhada e colaborativa. Em relação à comunicação via áudio, apesar de não ter ocorrido nenhum teste neste cenário, 93\% dos respondentes consideram importante essa forma de comunicação quando os programadores estão separados geograficamente.

Em relação ao processo de ensino/aprendizagem presencial 79\% dos respondentes concordam que a ferramenta contribui nesse sentido. Entre os que concordam, as justificativas mencionam o fato de que o professor não precisa se deslocar até o computador do aluno, outras alegam que as ferramentas do ambiente fornecem maior interatividade entre os usuários e o consideram como um facilitador.

\subsection{Cenário 2: Profissionais de Instituições de Ensino}

Neste estudo de caso, foram realizados experimentos com profissionais de desenvolvimento de software de três instituições de ensino superior do país: UFJF (Universidade Federal de Juiz de Fora/MG), UCS (Universidade de Caxias do Sul/RS) e FAI (Centro de Ensino Superior em Gestão, Tecnologia e Educação, de Santa Rita do Sapucaí/MG). Além da edição colaborativa e a comunicação utilizando mensagens de texto, neste estudo de caso foram feitos testes utilizando a comunicação através de áudio.

\subsubsection{O processo utilizado}

Neste cenário, cujo nível de dispersão é do tipo "Distância Nacional", conforme a classificação de [1], não houve nenhum contato pessoal para apresentação ou explicações detalhada das ferramentas do IdDE. Todos os contatos e instruções sobre a instalação e funcionamento do plugin foram mantidos através de e-mail e chat. Os testes ocorreram no mês de Fevereiro de 2011 e nenhum dos participantes utilizava o Netbeans em seu dia-a-dia.

Com a UCS, os testes foram realizados com a Coordenadora do Setor de Sistemas e as ferramentas testadas foram: edição colaborativa e chat. Já no caso da FAI, os testes ocorreram com a Coordenadora de TI e as ferramentas utilizadas foram: edição colaborativa, chat e comunicação via áudio. Na UFJF, os testes aconteceram com um Analista de Sistemas e as funcionalidades testa- 
das foram edição colaborativa, chat, áudio, ferramenta de tradução, gerenciamento de tarefas e agenda. Além disso, ocorreram testes internos entre dois analistas da UFJF.

Para a realização desses testes, o servidor XMPP utilizado foi o GMail e, para a comunicação através de áudio foi utilizado um servidor Asterisk ${ }^{1}$. Além da execução dos testes individuais com os representantes de cada instituição, ocorreu um teste conjunto com o analista de sistemas da UFJF e a Coordenadora de TI da FAI, momento no qual foram testadas as ferramentas de edição colaborativa, chat e áudio.

Para a realização destes testes, foi efetuada a instalação do servidor XMPP OpenFire ao invés do uso do servidor do GMail. Os testes realizados nestes cenários seguiram a mesma metodologia: primeiro eram feitas modificações nos códigos fontes e, posteriormente, eram confrontadas as versões finais dos documentos em cada ambiente para a verificação da consistência e integridade dos códigos.

\subsubsection{Análise dos resultados}

A avaliação dos testes neste cenário foi realizada por um total de quatro pessoas: uma da UCS, duas da UFJF e uma da FAI. Todos os participantes consideraram que a ferramenta auxiliou no processo de comunicação através de chat.

Da mesma forma todos os participantes avaliaram que o ambiente auxilia no processo de edição colaborativa de software. Entre as justificativas apresentadas está a simplicidade e a funcionalidade do ambiente e o fato de se poder acompanhar o local onde o usuário remoto está efetuando as modificações. Além disso, também foi citada a rapidez da atualização do arquivo remoto, muito superior se comparada ao uso os tradicionais aplicativos de acesso remoto como o compartilhamento de desktop. Por fim, foi mencionado que a edição compartilhada permite a prática de programação em pares do Extreme Programming de uma forma muito mais efetiva.

A ferramenta de áudio se mostrou útil e funcional para ambos os participantes que realizaram este teste. Igualmente, todos os participantes dos testes deste cenário consideram a comunicação via áudio importante num cenário onde os programadores estão separados geograficamente. Entre os motivos alegados, está o fato de que, se um programador precisar explicar as modificações feitas num código, é muito mais rápido e fácil que o chat textual

Em relação à contribuição da ferramenta para o processo de ensino/aprendizagem presencial, apenas um participante considerou que não tinha condições de avaliar a ferramenta sob este ponto de vista, por não ter utilizado ela para esse fim. Os demais consideraram que ela seja

${ }^{1}$ http://www.asterisk.org útil por auxiliar em diversas situações como, por exemplo, permitir que um professore corrija os programas dos alunos num laboratório, sem a necessidade de ficar se deslocando de computador em computador.

Em relação ao desenvolvimento distribuído de software, todos os participantes consideraram que a ferramenta quebra a barreira das distâncias, possibilitando que os usuários consigam trabalhar da mesma forma como se estivessem utilizando a ferramenta estando num mesmo ambiente.

$\mathrm{Na}$ opinião de todos os participantes, o fato do ambiente ter sido desenvolvido como módulo do Netbeans contribuiu para enriquecer sua a qualidade e praticidade funcional.

\subsection{Cenário 3: Professores Universitários}

Este estudo de caso foi realizado com quatro professores de cursos de graduação da área de tecnologia da informação, do Centro Universitário UNIVATES, na cidade de Lajeado/RS. Entre os professores, estavam os coordenadores dos cursos de Sistemas de Informação e Engenharia de Computação, além do Diretor do Centro de Ciências Exatas e Tecnológicas. As ferramentas testadas neste cenário foram: edição colaborativa e comunicação via chat.

\subsubsection{O processo utilizado}

A realização de testes junto a um grupo de professores teve por objetivo validar o potencial da ferramenta em ambientes de ensino presencial e à distância, sob o ponto de vista desses profissionais. Os professores envolvidos nos testes realizam atividades de EAD nas disciplinas que ministram. Os testes deste estudo de caso ocorreram no mês de Dezembro de 2010, no mesmo laboratório de informática utilizado anteriormente para os testes com os estudantes de graduação, descrito no Cenário 1.

Após breve apresentação do ambiente e suas funcionalidades, os professores instalaram o plugin no Netbeans e foram instruídos a se comunicarem utilizando o chat do IdDE e efetuassem a edição colaborativa de códigos fonte de programas.

Inicialmente a edição ocorreu aos pares, mas posteriormente todos os participantes passaram a editar o mesmo arquivo. Percebeu-se que não houve nenhuma perda de desempenho nesta circunstância e as modificações feitas por um professor eram enviadas aos demais quase que instantaneamente. Acredita-se que o fato de o servidor XMPP utilizado estar na rede própria instituição tenha contribuído para esse resultado.

\subsubsection{Análise de Resultados}

Neste grupo, todos os participantes concordam que o ambiente auxilia no processo de comunicação através de 
mensagens instantâneas. Da mesma forma, todos os participantes concordaram que a ferramenta é útil na edição colaborativa, afirmando que o sistema funcionou de forma satisfatória para alterações em um mesmo trecho de código, arbitrando na intervenção de diversos colaboradores.

Igualmente, consideraram que a ferramenta é extremamente útil em qualquer situação que demande desenvolvimento compartilhado: entre desenvolvedores de software, entre professor e aluno, na sala de aula ou auxílio à distância.

Em relação ao processo de ensino aprendizagem presencial, apenas um dos participantes considera que o ambiente até possa auxiliar, mas bem menos que no processo de aprendizagem à distância. Em contrapartida, os demais participantes consideram que o software seja interessante também nesse modelo, pois o professor pode acompanhar de forma mais efetiva o que os alunos estão fazendo. Avaliam que a ferramenta permitiria que o docente auxiliasse mais de um aluno simultaneamente, sem a necessidade de se deslocar na sala de aula, multiplicando o número de alunos atendidos no período de aula.

Os participantes foram unânimes ao afirmar que a ferramenta possui uma grande contribuição para a aprendizagem à distância. Entre as justificativas, está a contribuição para o auto aprendizado, capacidade inerente aos profissionais da informática. Aliado a isso, consideram que um recurso como esse seja extremamente útil para apoiar o ensino de programação à distância, afirmando que outros meios tradicionais como e-mail e chat são bem menos adequados, além de serem mais trabalhosos tanto para o professor quanto para o aluno. Com o uso do ambiente, o professor poderia explicar o funcionamento de determinado código para o aluno de forma online.

Segundo os participantes, a principal vantagem apontada foi a edição colaborativa de código. Afirmam que a ferramenta apresenta o resultado imediato das modificações, permitindo o acompanhamento e intervenção remota em tempo real e dentro do próprio ambiente de desenvolvimento. Além disso, consideram que o IdDE agiliza a atividade de desenvolvimento e o ensino e treinamento em programação, principalmente quando os participantes estão separados geograficamente, permitindo o auxílio e suporte a alunos e programadores menos experientes.

\section{Conclusões e Trabalhos Futuros}

O desenvolvimento de software geograficamente distribuído tem aumentado significativamente nos últimos anos e tornou-se uma necessidade de negócio para muitas corporações. Por outro lado, segundo Piaget, na medida em que o sujeito interage com, e sofre ação do objeto, ele vai produzindo sua capacidade de conhecer e vai produzindo também o próprio conhecimento.

Nesse sentido, este trabalho propõe um ambiente computacional que vai de encontro à teoria de Vygotsky, disponibilizando mecanismos onde o par mais capaz está presente, usando mecanismos de interação, coordenação e comunicação para a programação em pares distribuídos, potencializando o aprendizado.

Os resultados das avaliações dos estudos de caso comprovam que os objetivos traçados no planejamento deste trabalho foram plenamente atingidos. Na opinião de todos os participantes, as ferramentas de chat e edição colaborativa contribuem decisivamente para o desenvolvimento de software.

Da mesma forma, os participantes dos estudos de caso consideraram que as ferramentas do ambiente tornam possível sua utilização no processo de ensino/aprendizagem, tanto presencial quando à distância, bem como validam seu uso no desenvolvimento de software tanto por equipes locais, quando distribuídas.

Em relação à produtividade, $100 \%$ dos usuários consideraram que as ferramentas do ambiente contribuem para o seu aumento. No que se refere à utilização do Netbeans como base para a criação do aplicativo, todos os participantes avaliaram que tenha sido uma escolha apropriada, salientando que aplicativo desenvolvido agrega funcionalidades importantes para este IDE, tornando-o ainda mais completo.

Os resultados demonstram ainda que as escolhas dos protocolos XMPP e SIP se mostraram acertadas. Além de possibilitarem a interoperabilidade do ambiente com outros aplicativos e dispositivos, diversas avaliações fizeram referência à velocidade e rapidez das ferramentas do ambiente, principalmente se comparadas a ferramentas normalmente utilizadas no desenvolvimento de software, como compartilhamento de área de trabalho e acesso remoto aos computadores.

Durante o desenvolvimento do aplicativo percebeu-se que outros recursos interessantes poderiam ser incorporados ao ambiente. Da mesma forma, com a realização dos estudos de caso foram sugeridas várias funcionalidades a serem desenvolvidas em trabalhos futuros. Entre as funcionalidades, pode-se destacar:

- Implementar um algoritmo de controle e ordenação das mensagens, que garanta a unicidade de código para todos os participantes de uma sessão de edição colaborativa;

- Melhorar a apresentação das mensagens exibidas ao passar com o mouse sobre o ícone verde, que informam as alterações efetuadas num documento, agrupando as modificações por autor e possibilitando um acesso direto à janela do histórico das alterações; 
vídeo;

- Adicionar suporte a comunicação através de

- Desenvolver uma ferramenta de quadro branco, que permita que os usuários compartilhem imagens e desenhos, à exemplo do que ocorre com a edição colaborativa;

- Possibilitar a criptografia das mensagens que são enviadas de um ambiente para outro;

- Agrupar e integrar as janelas de chat ao ambiente do Netbeans;

- Portar o plugin para outros IDEs;

- Possibilitar a seleção do Codec de áudio a ser utilizado nas comunicações;

- Implementar uma ferramenta que possibilite a execução passo a passo todo o histórico de alterações de uma sessão de edição colaborativa, simulando todo o processo ocorrido anteriormente.

\section{Referencias Bibliográficas}

[1] J. Audy and R. Prikladnicki, Desenvolvimento Distribuído de Software. 1. ed. Rio de Janeiro: Elsevier, 2008.

[2] R. M. Borges, S. C. C. S. Pinto, J. L. V. Barbosa and D. N. F. Barbosa, Usando o modelo 3C de colaboração e Vygotsky no ensino de programação distribuída em pares. XVIII Simpósio Brasileiro de Informática na Educação (SBIE), 2007.

[3] E. Carmel, Global Software Teams - Collaborating Across Borders and Time Zones. Upper Saddle River, NJ: Prentice Hall, 1999. ISBN 0-13924218-X.

[4] E. Carmel, "Thirteen assertions for globally dispersed software development research", Proceedings of the Thirtieth Hawaii International Conference on System Sciences, vol.3, no.pp.445452 vol.3, 7-10, 1997.

[5] E. Carmel and R. Agarwal, "Tactical Approaches for Alleviating Distance in Global Software Development," IEEE Software, pp. 22-29, March/April, 2001.

[6] E. Carmel and P. Tjia, Offshoring Information Technology - Sourcing and Outsourcing to a Global Workforce. Cambridge, UK: Cambridge University Press, 2005.

[7] D. Damian and D. Moitra, "Global Software Development: How Far Have We Come?" IEEE Software, Vol. 23, No. 5, 2006.

[8] P. Dewan and J. Riedl, Toward computer- supported concurrent software engineering. Computer, vol.26, no.1pp.17-27, 1993.

[9] J. Davidson, J. Peters, M. Bhatia, S. Kalidindi and S. Mukherjee, Voice Over IP Fundamentals. 2. ed. Indianapolis, IN: Cisco Press, 2006.

[10] C. A. Ellis, S. J. Gibbs, and G. Rein, Groupware: some issues and experiences. ACM Communications Vol.34, No. 1, 39-58, 1991.

[11] S. R. K. Franco, O construtivismo e a educação. Porto Alegre: Mediação, 1996.

[12] H. Fuks, A. Raposo, M. Gerosa, "Do Modelo de Colaboração 3C à Engenharia de Groupware". Simpósio Brasileiro de Sistemas Multimídia e Web - WEBMIDIA, 2003.

[13] B. Hanks, "Student performance in CS1 with distributed pair programming". In Proceedings of the 10th Annual SIGCSE Conference on innovation and Technology in Computer Science Education. ITiCSE '05. ACM Press, New York, NY, 316-320, 2005.

[14] J. D. Herbsleb and R. E. Grinter, Splitting the Organization and Integrating the Code: Con-way's Law Revisited. In: ICSE'99, Los Angeles, CA. Proceeding, 1999.

[15] J. D. Herbsleb and D. Moitra, Global software development, IEEE Software, vol.18, no.2pp.1620, Mar/Apr 2001, 2001.

[16] C. Ho, S. Raha, E. Gehringer and L. Williams, "Sangam: a distributed pair programming plug-in for Eclipse". In Proceedings of the 2004 OOPSLA Workshop on Eclipse Technology Exchange. eclipse '04. ACM Press, New York, NY, 73-77, 2004.

[17] E. Lawrence, CollabEd: A Platform for Collaboratizing Existing Editors. International Confer-ence on Mobile, Hybrid, and On-line Learning, 2009.

[18] A. F. T. Primo and M. B. F. Cassol, Explorando o conceito de Intera-tividade: definições e taxionomias. In: Informática na Educação: teoria e prática. Porto Alegre: Revista PGIE/UFRGS, v.2, n.2, out, 1999.

[19] P. Saint-Andre, K. Smith and R. Tronçon, XMPP: The Definitive Guide - Building Real-Time Applications with Jabber Technologies. 1. ed. Sebastopol, CA: O'Reilly Media, 2009.

[20] T. Schummer and J. Schummer, Support for Distributed Teams in Extreme Programming. Extreme Programming Examined, Succi, G., Mar- 
chesi, M. eds., p. 355-377, Boston, MA: Addison Wesley, 2001.

[21] H. Sinnreich and A. B. Johnston, Internet Communications Using SIP: Delivering VoIP and Multimedia Services with Session Initiation Protocol. 2. ed. Indianapolis, IN: Wiley Publish-ing, 2006.

[22] D. Stotts, L. Williams, N. Nagappan, P. Beheti, D. Jen and A. Jackson, Virtual Teaming: Experiments and Experiences with Distributed Pair Programming, Proceedings of the Third XP Agile Universe Conference, 2003.

[23] D. F. G. Trindade, T. F. C. Tait and E. H. M. Huzita, A tool for supporting the communication in distributed software development environ-ment. Journal of Computer Science \& Technology, 8 (2), pp: 118-124, 2008.

[24] L. A.Williams and R. R. Kessler, All I really need to know about pair programming I learned in kindergarten. ACM Communications. Vol. 43, No. 5, 108-114, 2000.

[25] Juan Manuel Adán-Coello, Wiris Serafim de Menezes, Eustáquio São José de Faria, Carlos Miguel Tobar, Conflito Sócio-Cognitivo e Estilos de Aprendizagem na Formação de Grupos para o Aprendizado Colaborativo de Programação de Computadores, Revista Brasileira de Informática na Educação, RBIE, V.16. N. 03, 2008. 